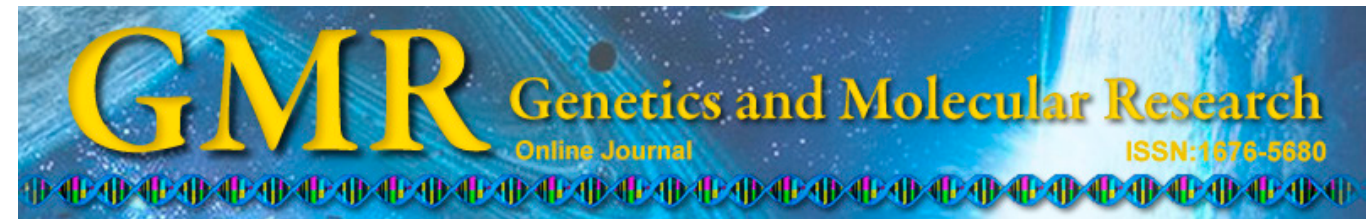

\title{
Genetic divergence for high-molecular weight glutenin subunits $(H M W-G S)$ in indigenous landraces and commercial cultivars of bread wheat of Pakistan
}

\author{
F. Yasmeen ${ }^{1}$, H. Khurshid ${ }^{2}$ and A. Ghafoor ${ }^{2}$ \\ ${ }^{1}$ Department of Biochemistry, Quaid-i-Azam University, Islamabad, Pakistan \\ ${ }^{2}$ Plant Genetic Resources Institute, National Agricultural Research Center, \\ Islamabad, Pakistan \\ Corresponding author: A. Ghafoor \\ E-mail: ghafoor59pk@yahoo.com
}

Genet. Mol. Res. 14 (2): 4829-4839 (2015)

Received July 18, 2014

Accepted April 28, 2015

Published May 11, 2015

DOI http://dx.doi.org/10.4238/2015.May.11.15

\begin{abstract}
Wheat flour quality is an important consideration in the breeding and development of new cultivars. A strong association between high-molecular weight glutenin subunits $(H M W-G S)$ and bread making quality has resulted in the widespread utilization of $H M W-G S$ in wheat breeding. In this study, we analyzed 242 lines of wheat, including landraces from the provinces of Punjab and Baluchistan, as well as the commercial varieties of Pakistan, to determine allelic variation in the $G l u-A 1$, $G l u-B 1$, and Glu-D1 loci encoding $H M W-G S$. Higher genetic diversity was observed for $H M W-G S$ in landraces from Baluchistan, followed by landraces collected from Punjab and then commercial varieties. Rare and uncommon subunits were observed in $G l u-B 1$, whereas $G l u$-Al was less polymorphic. However, Glu-B1 was the highest contributor to overall diversity (78\%), with a total of 31 rare alleles, followed by Glu-D1 $(20 \%)$ with the high quality $5+10$ allele and other variants. Commercial cultivars possessed favorable alleles, potentially from indirect selection for wheat flour quality by the breeders; however, this indirect selection
\end{abstract}


has decreased the pedigree base of commercial cultivars. The allelic combinations, including $2 *, 5+10$, and $17+18$, showing high quality scores were frequent among landraces, indicating their usefulness in future crop improvement and breeding programs.

Key words: Bread making quality; Glu-A, Glu-B, Glu-D1; Triticum aestivum

\section{INTRODUCTION}

The genetic diversity for high-molecular weight glutenin subunits $(H M W-G S)$ has been frequently utilized for wheat improvement because of its relationship with bread making quality, high polymorphism (Payne and Lawrence, 1983; Degaonkar et al., 2005; Goutam et al., 2013), low price, and easy evaluation as compared to morphological evaluation and the use of other molecular markers (Yu et al., 2013). Gluten is major viscoelastic protein present in mature wheat grains and accounts for $80-85 \%$ of the total protein content in flour (Li et al., 2009). Gliadins and glutenins are components of gluten that significantly influence the utilization of grain in food processing (Peter et al., 2002). The composition and amount of these components, particularly $H M W-G S$, are associated with dough making quality and baking quality of wheat flour (Li Vigni et al., 2013). $H M W-G S$ differs from low-molecular weight glutenin subunits through variations in amino acid composition and molecular weight, as the former group ranges in size from 77-160 $\mathrm{kDa}$, while the latter are $23-68 \mathrm{kDa}$ (Žilić et al., 2011). Allelic variation in $H M W-G S$ contributes to $45-70 \%$ of the variation in bread making quality of some European lines (Payne, 1987) and has also been documented in Pakistani wheat varieties (Sultana et al., 2007).

The genes for $H M W-G S$ are located at 3 loci, including Glu-A1, Glu-B1, and Glu-D1 on the long arms of homologous group-1 chromosome of each group (Payne, 1987). Previous studies confirmed the presence of 2 tightly-linked genes, Glu 1-1 and Glu 1-2, at each Glu1 locus (Glu-A1, Glu-B1, and Glu-D1) encoding the $x$ - and $y$-type subunits, respectively. Therefore, in common wheat, $1 A x$ or null subunits are encoded by Glu-A1,1Bx, and $1 B y$ subunits by Glu-B1, while the $1 D x$ and $1 D y$ subunits are encoded by the Glu-D1 locus (Payne and Lawrence, 1983; Payne, 1987). Electrophoretic analysis revealed that $x$-type subunits have high molecular weight $(80-88 \mathrm{kDa})$ and low mobility, while $y$-type subunits have low molecular weight (67-73 kDa) and high mobility (Yan et al., 2009). Payne and Lawrence (1983) published the catalogue of Glu-1 alleles and reported 3 alleles (Null, 1 and $2^{*}$ ) at the Glu-Al locus, 11 alleles $(7,20,21,22,7+8,7+9,6+8,13+16,13+19,14+15$, and $17+18)$ at the $G l u-B 1$ locus, and 6 alleles $(2+12,3+12,4+12,5+10,2+10$, and $2.2+12)$ at the Glu-Dl locus. McIntosh et al. (2003) reported 22 alleles at the Glu-A1 locus, 56 alleles at the Glu-B1 locus, and 37 alleles at the Glu-D1 locus. However, more than 20 different $H M W-G S$ genes have been identified and characterized. Some subunits, particularly $5+10$ at the Glu-D1 locus and the $14+15$ subunit pair at $G l u-B 1$ are known to be significantly associated with greater gluten viscoelasticity and strong dough characteristics (Redaelli et al., 1997; Liu et al., 2005). In this study, we evaluated the genetic variation at Glul loci linked with $H M W-G S$ among indigenous wheat landraces collected from the provinces of Baluchistan and Punjab, as well as in commercial varieties of Pakistani origin to identify desirable alleles responsible for good bread-making quality of flour. The landraces are not common in the province of Punjab, whereas a predominance of landraces has been reported in the province of Baluchistan (Ahmad et al., 2013); thus, we 
compared diversity patterns of $H M W-G S$ for landraces collected from the Punjab and Baluchistan provinces. Those from the Punjab province contribute to the diversity of many cereals and legumes (Khan et al., 2013), and thus landraces from this area were collected and included in the present study to increase the understanding of Pakistani landraces.

\section{MATERIAL AND METHODS}

Among the 242 wheat lines used for biochemical analysis in the present study, 51 landraces were collected from Punjab, 122 landraces from Baluchistan, and 69 were commercial varieties. Sample collection strictly involved collection of only landraces from both provinces; the lower number of landraces from Punjab was because of the adaptation of improved cultivars in this province, whereas indigenous landraces are commonly cultivated in the province of Baluchistan, and thus a larger number was collected. Prior to the analysis, all 242 lines were self-crossed for 2 consecutive generations to ensure homozygosity. The germplasms evaluated in the present study were preserved in the gene bank of the Plant Genetic Resources Institute, National Agriculture Research Centre Islamabad. For protein extraction, the half seeds (excluding the embryo) were ground to a fine powder using a mortar and pestle. Next, $400 \mu \mathrm{L}$ protein extraction buffer was added to $0.01 \mathrm{~g}$ seed flour in an Eppendorf tube and mixed thoroughly using a needle. The protein extraction buffer was composed of 0.6057 $\mathrm{g}$ Tris, $0.2 \mathrm{~g}$ sodium dodecyl sulfate (SDS), $30.3 \mathrm{~g}$ urea, $1 \mathrm{~mL}$ 2-mercaptoethanol, a small amount of bromophenol blue, $70 \mathrm{~mL}$ distilled water, and $\mathrm{HCl}$ (adjusted to $\mathrm{pH} 8.0$ ). Bromophenol blue was used as a tracking dye for the movement of protein in the gel. Ten samples from each line were analyzed using SDS-polyacrylamide gel electrophoresis. The samples were mixed thoroughly by vortexing and centrifuged at $12054 \mathrm{~g}$ for $10 \mathrm{~min}$, and then stored at $4^{\circ} \mathrm{C}$ until electrophoresis (less than 1 week).

To prepare the $11.5 \%$ separation gel, $5 \mathrm{~mL}$ solution A (36.3 g Tris, $0.4 \mathrm{~g}$ SDS, 70 $\mathrm{mL}$ distilled water, and $\mathrm{HCl}$ to adjust the $\mathrm{pH}$ to 8.8 ) was mixed with $7.6 \mathrm{~mL}$ solution $\mathrm{C}(30 \mathrm{~g}$ acrylamide, $0.8 \mathrm{~g}$ bis-acrylamide, and distilled water to make a volume of $100 \mathrm{~mL}$ ), which was followed by the addition of $7.4 \mathrm{~mL}$ distilled water. Next, $200 \mu \mathrm{L} 10 \%$ ammonium persulfate and $15 \mu \mathrm{L}$ N,N,N',N'-tetra methylethylenediamine were added. The separation gel solution was poured into the space between 2 glass plates (to $2 \mathrm{~cm}$ from the top). Next, $120 \mu \mathrm{L}$ distilled water was added to the top of the separation gel. The gel was polymerized for $30 \mathrm{~min}$. The $4.5 \%$ stacking gel was prepared by mixing $2.5 \mathrm{~mL}$ solution B (5.98\% Tris, $0.4 \mathrm{~g}$ SDS, and 80 $\mathrm{mL}$ distilled water, and the $\mathrm{pH}$ was adjusted to $\mathrm{pH} 7.0$ using $\mathrm{HCl}$, and total volume was 100 $\mathrm{mL}$ ) with $1.5 \mu \mathrm{L}$ solution $\mathrm{C}$. Next, $70 \mu \mathrm{L} 10 \%$ ammonium persulfate and $17 \mu \mathrm{L}$ N,N,N',N'tetra methylethylenediamine were added. The distilled water was removed from the top of the separation gel, followed by the pouring of the stacking gel solution onto the separation gel. Comb clips were fixed and the gels were polymerized.

Electrode buffer solution (3.0 g Tris, $14.4 \mathrm{~g}$ glycine, $1.25 \mathrm{~g} \mathrm{SDS}$, and distilled water to make a total volume of $1 \mathrm{~L}$ ) was poured into the bottom tank of the gel apparatus. The comb and gasket seal were removed and the gel plates were fixed in the apparatus. The electrode buffer solution was poured into the upper tank. Next, $6 \mu \mathrm{L}$ protein supernatant was loaded into the wells of the gel using a micropipette. The current was maintained at $100 \mathrm{~mA}$ and electrophoresis was performed until the bromophenol blue (blue line) reached the bottom of the gel (approximately $3.5 \mathrm{~h}$ ). Three standards (Chinese Spring, Pavon, C 591) containing known $H M W$ $G S$ were included for comparison and data recording. After electrophoresis, the gels were 
stained using a staining solution (2.25 g Coomassie brilliant blue R250, $440 \mathrm{~mL}$ methanol, 60 $\mathrm{mL}$ acetic acid, and $500 \mathrm{~mL}$ distilled water). The gels were shaken in the staining solution for $25 \mathrm{~min}$. Destaining was carried out in a destaining solution $(200 \mathrm{~mL}$ methanol, $50 \mathrm{~mL}$ acetic acid, and $750 \mathrm{~mL}$ distilled water). Gels were shaken gently until the background color had disappeared and the electrophoretic bands were clearly visible. The destained gel was stored in distilled water in a refrigerator and used for data recording. The gels were scanned and images were acquired. The presence of a band was scored as ' 1 ' and the absence of a band as ' 0 ' in a binary fashion. The data were analyzed for cluster analysis using the unweighted pair group method with arithmetic mean with the STATISTICA 7.0 software for Windows 7 (StatSoft, Inc., Tulsa, OK, USA). To avoid effects resulting from differences in scale, the means of each trait were standardized prior to cluster analysis for quantitative data.

\section{RESULTS}

The allelic frequency and percent polymorphism are presented in Table 1. Landraces collected from Baluchistan showed the largest number of subunits, while commercial cultivars showed the lowest. Six subunits $\left(2,3,8^{* *}, 15,18^{* *}\right.$, and 20) were absent among the commercial cultivars and landraces from Punjab. Among all 3 loci, $2 \%$ allelic polymorphism was attributed by the A genome, $78 \%$ by the B genome, and $20 \%$ by the $\mathrm{D}$ genome. Considerable allelic variation in $H M W-G S$ was observed among the 242 wheat lines with varying degrees of allelic frequencies from landraces from Punjab and Baluchistan and commercial cultivars for all 3 loci (Glu-A1, Glu$B 1$, and $G l u-D 1$ ). Four alleles were observed in Glu-A1; among these, 1 was polymorphic. The largest number of polymorphisms was present in $G l u-B 1$, as 31 polymorphic subunits were found among the 59 subunits (Table 1). The Glu-Dl also carried a large number of polymorphisms, including 8 rare alleles of 16 alleles. At the Glu-Al locus, 3 allelic variants, a, b, and c, or 1, 2*, and Null, were observed. However, an unusual 2 ' allele was present in the landrace with accession number " 11162 " of Baluchistan origin. The frequency distribution for the 1,2*, and Null alleles in the landraces collected from Punjab was 17.6\% (9 landraces), 33.33\% (17 landraces), and 47\% (25 landraces), respectively. The Null type allele was abundant (60.6\%) in the landraces collected from Baluchistan, preceded by the Glu-Al "a" or 1 allele (22\%) and $2 *$ allele $(16.39 \%)$.

The commercial varieties contained the $2 *$ allele in 38 varieties $(55 \%)$ followed by 1 and Null type allele, accounting for 25 and $20 \%$, respectively. Among the landraces collected from Baluchistan, maximum allelic variation was found at the Glu-B1 locus, where 30 different alleles were reported, while landraces from Punjab contained 19 subunits or pairs of subunits at this locus. In contrast, 10 alleles were recorded at $G l u-B 1$ for commercial varieties. Cluster-wise allelic frequencies and quality scores for landraces from Baluchistan, landraces from Punjab, and in commercial cultivars are shown in Tables 2a-2c, respectively. For the landraces originating from Punjab, the subunit $17+18$ was present in 21 accessions $(41.17 \%)$, and the single allele 7 was present in 8 accessions (15.68\%). Subunit $17+18$ is considered to be significant for dough making quality (Table 2a). The other 17 alleles, including single subunits 16 and 9 , were distributed among $<4 \%$ landraces. Among commercial varieties, subunit $17+18$ was observed in 25 commercial varieties with a $36.23 \%$ frequency distribution, which may be related to selection pressure in the breeding of wheat for quality. Alleles $7+9$ and $7^{*+9}$ were found in $21(30.43 \%)$ and $13(18.84 \%)$ varieties, respectively. Alleles $7^{* *+9}, 7+8$, $13+16,7 *(13+16), 7 *+8$, and $7+814$ were found in single genotypes, conferring unique allelic combinations. At the Glu-D1 locus, 4 subunits $2+12(80 \%), 5+10(15.68 \%), 12(1.96 \%)$, and 
$5(1.96 \%)$ were observed among landraces from Punjab. Baluchistan accessions were found to have the highest diversity at this locus, as 9 different alleles were present at Glu-D1 (Table 2 b). Allele $2+12$ was predominant in 69 landraces $(56.55 \%)$, followed by $5+10$ in 34 landraces $(27.86 \%)$. Allelic variants $2+12 *$ and 12 were observed in $6(4.91 \%)$ and $4(3.27 \%)$ landraces, respectively. Subunits $10,3+12,12 *, 5+12 *$, and $5+12$ were rare, with low frequency distributions $(0.8-2.45)$. The commercial varieties carried less variation at the GluD1 locus, as only 3 alleles " $s ", ~ " ~ y "$ ", and " $x$ " or $2+12,5+10$, and a single subunit of 12 were observed. Both $2+12$ and 5+10 were present among 34 lines, with frequency distributions of $49.2 \%$ each, while allele 12 was present only in Chakwal 97 (Table 2c).

Table 1. Allelic frequency of HMW-GS in bread wheat landraces and commercial varieties.

\begin{tabular}{|c|c|c|c|c|c|c|c|c|c|c|}
\hline \multirow[t]{2}{*}{$H M W-G S$} & \multicolumn{2}{|c|}{ Commercial cultivars } & \multicolumn{2}{|c|}{$\begin{array}{c}\text { Landraces } \\
\text { from Baluchistan }\end{array}$} & \multicolumn{2}{|c|}{$\begin{array}{l}\text { Landraces } \\
\text { from Punjab }\end{array}$} & \multicolumn{2}{|c|}{$\begin{array}{l}\text { Checks (Chinese spring, } \\
\text { Pavon, C 591) }\end{array}$} & \multicolumn{2}{|c|}{ Combined } \\
\hline & $f$ & Percent & $\bar{f}$ & Percent & $\bar{f}$ & Percent & $f$ & Percent & $f$ & Percent \\
\hline Null & 14 & 20.3 & 75 & 61.5 & 24 & 47.1 & 1 & 33.3 & 114 & 47.1 \\
\hline 1 & 17 & 24.6 & 27 & 22.1 & 9 & 17.6 & 1 & 33.3 & 54 & 22.3 \\
\hline 2 & 34 & 49.3 & 78 & 63.9 & 41 & 80.4 & 2 & 66.6 & 155 & 64.0 \\
\hline $2 *$ & 38 & 55.1 & 20 & 16.4 & 17 & 33.3 & 1 & 33.3 & 76 & 31.4 \\
\hline 2 & 0 & 0.0 & 1 & 0.8 & 0 & 0.0 & 0 & 0.0 & 1 & 0.4 \\
\hline 3 & 0 & 0.0 & 1 & 0.8 & 0 & 0.0 & 0 & 0.0 & 1 & 0.4 \\
\hline 5 & 34 & 49.3 & 35 & 28.7 & 9 & 17.6 & 1 & 33.3 & 79 & 32.6 \\
\hline 6 & 0 & 0.0 & 3 & 2.4 & 3 & 5.9 & 0 & 0.0 & 6 & 2.5 \\
\hline 7 & 27 & 39.1 & 65 & 53.3 & 13 & 25.5 & 1 & 33.3 & 106 & 43.8 \\
\hline $7^{*}$ & 15 & 21.7 & 9 & 7.4 & 5 & 9.8 & 0 & 0.0 & 29 & 11.9 \\
\hline $7 * *$ & 1 & 1.5 & 23 & 18.9 & 6 & 11.7 & 0 & 0.0 & 30 & 12.4 \\
\hline 8 & 5 & 7.3 & 26 & 21.3 & 8 & 15.7 & 1 & 33.3 & 40 & 16.5 \\
\hline $8^{*}$ & 1 & 1.5 & 7 & 5.7 & 1 & 1.9 & 0 & 0.0 & 9 & 3.7 \\
\hline $8 * *$ & 0 & 0.0 & 8 & 6.6 & 0 & 0.0 & 0 & 0.0 & 8 & 3.3 \\
\hline 9 & 35 & 50.7 & 38 & 31.2 & 7 & 13.7 & 0 & 0.0 & 80 & 33.1 \\
\hline 10 & 35 & 50.7 & 36 & 29.5 & 8 & 15.7 & 1 & 33.3 & 80 & 33.1 \\
\hline 12 & 34 & 49.3 & 75 & 61.4 & 41 & 80.4 & 2 & 66.7 & 152 & 62.8 \\
\hline $12^{*}$ & 0 & 0.0 & 8 & 6.5 & 1 & 1.9 & 0 & 0.0 & 9 & 3.7 \\
\hline 13 & 2 & 2.9 & 5 & 4.1 & 2 & 3.92 & 1 & 33.3 & 10 & 4.1 \\
\hline 14 & 1 & 1.5 & 8 & 6.6 & 0 & 0.0 & 0 & 0.0 & 9 & 3.7 \\
\hline $14^{*}$ & 0 & 0.0 & 0 & 0.0 & 1 & 1.9 & 0 & 0.0 & 1 & 0.4 \\
\hline 15 & 0 & 0.0 & 8 & 6.6 & 0 & 0.0 & 0 & 0.0 & 8 & 3.3 \\
\hline 16 & 2 & 2.9 & 3 & 2.5 & 3 & 5.9 & 1 & 33.3 & 9 & 3.7 \\
\hline 17 & 25 & 36.2 & 14 & 11.5 & 22 & 43.1 & 1 & 33.3 & 62 & 25.6 \\
\hline 18 & 25 & 36.2 & 13 & 10.6 & 22 & 43.1 & 1 & 33.3 & 61 & 25.2 \\
\hline $18^{* *}$ & 0 & 0.0 & 2 & 1.6 & 0 & 0.0 & 0 & 0.0 & 2 & 0.8 \\
\hline 20 & 0 & 0.0 & 1 & 0.8 & 0 & 0.0 & 0 & 0.0 & 1 & 0.4 \\
\hline 22.1 & 1 & 1.4 & 0 & 0.0 & 0 & 0.0 & 0 & 0.0 & 1 & 0.4 \\
\hline
\end{tabular}

Quality scores were assigned to each subunit band produced by alleles at the Glu1 loci of chromosomes A, B, and D, as defined by Payne et al. (1987). The list of different allelic combinations and frequency distributions are shown in Table 1. Unique or rare alleles were present in the population; as in Punjab accessions, at Glu-B1, subunits 16, 14*+9, 9(17+18), $7 * *+8^{*}, 6+7,8(13+16), 6+9$ and $7 *(7 * *)+8$ were resolved besides single subunit 5 at GluD1. Accessions from Baluchistan presented the highest number of rare subunits, including $2^{\prime}$ at GluAl and $7 *+8(8 * *), 7(7 *)+9,8^{*}, 7+8^{*}, 7+8^{* *}, 7 * *+9,7(7 * *)+9,13,7 * *+8 * *$, $8^{* *}(17+18), 14+15,6(14+15), 7(14+15), 20,7(7 *)+8,\left(8^{*}, 7+9\right), 8^{*}(7 *+9), 6(17+18)$, and 17 at Glu-B1, and $3+12,2+12 *, 10,12 *, 5+12 *$, and $5+12$ at $G l u-D 1$, which were absent in the commercial varieties and Punjab based accessions. Commercial varieties exhibited unique allelic variants only at $G l u-B 1$, including $7 * *+9,7+8(8 *), 14$, and $7 *(13+16)$. 
Table 2. Cluster-wise allelic frequency and quality scores of landraces of bread wheat collected from Punjab and Baluchistan provinces.

\begin{tabular}{|c|c|c|c|c|c|c|c|c|c|c|}
\hline \multicolumn{2}{|c|}{ A. Punjab Province } & \multirow[t]{2}{*}{$f$} & \multicolumn{4}{|c|}{ Allelic frequency } & \multicolumn{3}{|c|}{ Quality score } & \multirow[t]{2}{*}{ Total } \\
\hline & & & $\overline{\text { Glu-Al }}$ & & Ilu-Bl & $\overline{G l u-D 1}$ & $\overline{G l u-A l}$ & Glu-BI & $\overline{G l u-D 1}$ & \\
\hline 1 & 11364 & 1 & $2 *$ & & 16 & 12 & 3 & $?$ & $?$ & $3+?$ \\
\hline 2 & 11360 & 1 & $2^{*}$ & & $14 *+9$ & $2+12$ & 3 & ? & 2 & $<10$ \\
\hline 3 & 11348 & 1 & $\mathrm{~N}$ & & $(17+18)$ & $2+12$ & 1 & ? & 2 & $<10$ \\
\hline 4 & $11349,18669,18673,8677,18687,18690$ & 6 & $\mathrm{~N}$ & & $7+18$ & $2+12$ & 1 & 3 & 2 & 6 \\
\hline 5 & $\begin{array}{c}11162,11363,18672,18678,18679 \\
18681,18682,18684,18685 \\
18689,18694,18702,18703\end{array}$ & 13 & $2^{*}$ & & $17+18$ & $2+12$ & 3 & 3 & 2 & 8 \\
\hline 6 & 11356 & 1 & 1 & & $17+18$ & $2+12$ & 3 & 3 & 2 & 8 \\
\hline 7 & 11350,18670 & 2 & $\mathrm{~N}$ & & $* *+8$ & $2+12$ & 1 & ? & 2 & $<10$ \\
\hline 8 & 11355 & 1 & $\mathrm{~N}$ & & $7 * *$ & $2+12$ & 1 & ? & 2 & $<10$ \\
\hline 9 & 18707 & 1 & $\mathrm{~N}$ & & $* *+8 *$ & $2+12$ & 1 & ? & 2 & $<10$ \\
\hline 10 & $11351,18676,18688,18693,18706$ & 5 & $\mathrm{~N}$ & & 7 & $2+12$ & 1 & 1 & 2 & 4 \\
\hline 11 & 11359 & 1 & $\mathrm{~N}$ & & & $2+12$ & 1 & ? & 2 & $<10$ \\
\hline 12 & 18680,18697 & 2 & $\mathrm{~N}$ & & $7+8$ & $2+12$ & 1 & 3 & 2 & 6 \\
\hline 13 & 18683 & 1 & $2^{*}$ & & $\left(7^{* *}\right)$ & $2+12$ & 3 & ? & 2 & $<10$ \\
\hline 14 & 18704 & 1 & $2^{*}$ & & $6+7$ & $2+12$ & 3 & ? & 2 & $<10$ \\
\hline 15 & 18695 & 1 & $\mathrm{~N}$ & & 7 & $2+12$ & 1 & 1 & ? & $<10$ \\
\hline 16 & 11352,11353 & 2 & $\mathrm{~N}$ & & $7^{*+9}$ & $2+12$ & 1 & 2 & 2 & 5 \\
\hline 17 & 18698. 18699 & 2 & $\mathrm{~N}$ & & $7^{*+8}$ & $2+12$ & 1 & 3 & 2 & 6 \\
\hline 18 & 18696 & 1 & $\mathrm{~N}$ & & $(13+16)$ & 5 & 1 & ? & ? & $<10$ \\
\hline 19 & 18701 & 1 & 1 & & $3+16$ & $5+10$ & 3 & 3 & 4 & 10 \\
\hline 20 & 11361 & 1 & 1 & & $17+18$ & $5+10$ & 3 & 3 & 4 & 10 \\
\hline 21 & 18674 & 1 & 1 & & 9 & $5+10$ & 3 & ? & 4 & $7+?$ \\
\hline 22 & 18692 & 1 & 1 & & $7+9$ & $5+10$ & 3 & 2 & 4 & 9 \\
\hline 23 & 18705 & 1 & 1 & & $6+9$ & $5+10$ & 3 & ? & 4 & $7+?$ \\
\hline 24 & 18675 & 1 & 1 & & $6+7$ & $5+10$ & 3 & ? & 4 & $7+?$ \\
\hline 25 & 18700 & 1 & 1 & & 7 & $5+10$ & 3 & ? & 4 & $7+?$ \\
\hline 26 & 18780 & 1 & 1 & & $(7 * *)+8$ & $5+10$ & 3 & ? & 4 & $7+?$ \\
\hline \multirow{2}{*}{\multicolumn{3}{|c|}{ B. Baluchistan Province }} & \multicolumn{4}{|c|}{ Allelic frequency } & \multicolumn{3}{|c|}{ Quality score } & \multirow[t]{2}{*}{ Total } \\
\hline & & & & $l u-A I$ & Glu-B1 & $\overline{G l u-D 1}$ & Glu-Al & Glu-Bl & $\overline{G l u-D 1}$ & \\
\hline 1 & \multicolumn{2}{|l|}{11145} & 1 & $2 *$ & $7^{*+8}$ & $2+12$ & 3 & 3 & 2 & 8 \\
\hline \multicolumn{3}{|c|}{11333} & 1 & 1 & $7 *+8\left(8^{* *}\right)$ & $2+12$ & 3 & ? & 2 & $<10$ \\
\hline \multirow{2}{*}{\multicolumn{3}{|c|}{$\begin{array}{l}11150 \\
11154\end{array}$}} & 1 & $2^{*}$ & $7+8$ & $3+12$ & 3 & 3 & 2 & 8 \\
\hline & & & 1 & $\mathrm{~N}$ & $7+8$ & $2+12$ & 1 & 3 & 2 & 6 \\
\hline 5 & \multicolumn{2}{|c|}{$\begin{array}{l}1,11189,11190,11209,11212 \\
71,11286,11309,11327,11536\end{array}$} & 14 & $\mathrm{~N}$ & $7+8$ & $2+12$ & 1 & 3 & 2 & 6 \\
\hline \multirow{2}{*}{\multicolumn{3}{|c|}{$\begin{array}{l}11238 \\
11280\end{array}$}} & 1 & $2 *$ & 7 & $2+12$ & 3 & 1 & 2 & 6 \\
\hline & & & 1 & $2^{*}$ & $7+8$ & $2+12$ & 3 & 3 & 2 & 8 \\
\hline 8 & $\begin{array}{l}11177,11227,11228,11229,11248,11276 \\
11283,11284,11285,11305,11317,11323\end{array}$ & & 12 & $\mathrm{~N}$ & $7+9$ & $2+12$ & 1 & 2 & 2 & 5 \\
\hline 9 & $\begin{array}{c}11226.11235,11236,11239,11240,11242 \\
11244,11255,11259,11278,11293\end{array}$ & & 11 & $\mathrm{~N}$ & 7 & $2+12$ & 1 & 1 & 2 & 4 \\
\hline 10 & 11304 & & 1 & $\mathrm{~N}$ & $7\left(7^{*}\right)+9$ & $2+12$ & 1 & ? & 2 & $<10$ \\
\hline 11 & 11237 & & 1 & $\mathrm{~N}$ & $8^{*}$ & $2+12$ & 1 & ? & 2 & $<10$ \\
\hline 12 & $11281,11329,11334$ & & 3 & $\mathrm{~N}$ & $7+8^{*}$ & $2+12$ & 1 & 3 & 2 & 6 \\
\hline 13 & 11158,11306 & & 2 & $\mathrm{~N}$ & $7+8^{* *}$ & $2+12$ & 1 & ? & 2 & $<10$ \\
\hline 14 & 11223 & & 1 & $2 *$ & $7+8 * *$ & $2+12$ & 3 & $?$ & 2 & $<10$ \\
\hline 15 & $11221,11243,11246$ & & 3 & $\mathrm{~N}$ & $7 * *$ & $2+12$ & 1 & ? & 2 & $<10$ \\
\hline 16 & $11263,11338,11344$ & & 3 & $\mathrm{~N}$ & $7^{* *+9}$ & $2+12$ & 1 & ? & 2 & $<10$ \\
\hline 17 & 13191 & & 1 & $\mathrm{~N}$ & $7^{* *+8}$ & $2+12$ & 1 & ? & 2 & $<10$ \\
\hline 18 & $11294,11295,11296,11297,11302$ & & 5 & $2^{*}$ & $7(7 * *)+9$ & $2+12$ & 3 & ? & 2 & $<10$ \\
\hline 19 & 11312 & & 1 & $2^{*}$ & $7+9$ & $2+12$ & 3 & 2 & 2 & 7 \\
\hline 20 & 11161 & & 1 & $\mathrm{~N}$ & $?$ & $?$ & 1 & ? & ? & $?$ \\
\hline 21 & 11193 & & 1 & $\mathrm{~N}$ & 13 & $2+12$ & 1 & ? & 2 & $<10$ \\
\hline 22 & 11531 & & 1 & 1 & 13 & 12 & 3 & ? & $?$ & $3+?$ \\
\hline 23 & 11156 & & 1 & $2^{*}$ & $7 * *+8 * *$ & $2+12$ & 3 & ? & 2 & $<10$ \\
\hline 24 & 11315 & & 1 & $2 *$ & $7 * *+8 * *$ & $2+12 *$ & 3 & ? & $?$ & $3+?$ \\
\hline 25 & 11261 & & 1 & $2 *$ & $7\left(7^{* *}\right)$ & $2+12 *$ & 3 & ? & ? & $3+?$ \\
\hline 26 & 11272 & & 1 & $\mathrm{~N}$ & $7 * *$ & $2+12 *$ & 1 & ? & ? & $1+?$ \\
\hline 27 & 11224 & & 1 & $2^{*}$ & $17+18$ & $2+12$ & 3 & 3 & 2 & 8 \\
\hline
\end{tabular}


Table 2. Continued.

B. Baluchistan Province

\begin{tabular}{|c|c|c|c|c|c|c|c|c|c|}
\hline & & \multirow[t]{2}{*}{$f$} & \multicolumn{3}{|c|}{ Allelic frequency } & \multicolumn{3}{|c|}{ Quality score } & \multirow[t]{2}{*}{ Total } \\
\hline & & & Glu-Al & Glu-B1 & Glu-D1 & Glu-Al & Glu-B1 & Glu-D1 & \\
\hline 28 & 11528 & 1 & $\mathrm{~N}$ & $17+18$ & $2+12$ & 1 & 3 & 2 & 6 \\
\hline 29 & 11335 & 1 & $\mathrm{~N}$ & $8^{* *}, 17+18$ & $2+12$ & 1 & ? & 2 & $<10$ \\
\hline 30 & 11325 & 1 & $2 *$ & $17+18$ & $2+12 *$ & 3 & 3 & $?$ & $6+?$ \\
\hline 31 & 11162 & 1 & $2^{\prime}$ & $7+8$ & 10 & $?$ & 3 & $?$ & $3+?$ \\
\hline 32 & 11155,11178 & 2 & $\mathrm{~N}$ & $14+15$ & $2+12 *$ & $i$ & 2 & ? & $3+?$ \\
\hline 33 & 11186 & 1 & $\mathrm{~N}$ & $14+15$ & $2+12$ & 1 & 2 & 2 & 5 \\
\hline 34 & 11299 & 1 & $\mathrm{~N}$ & $6,14+15$ & $12^{*}$ & 1 & ? & $?$ & $1+?$ \\
\hline 35 & 11157 & 1 & $2 *$ & $9,14+15$ & 12 & 3 & ? & ? & $3+?$ \\
\hline 36 & 11183 & 1 & $2 *$ & $14+15$ & 12 & 3 & 2 & ? & $5+?$ \\
\hline 37 & 11164 & 1 & $\mathrm{~N}$ & $7,14+15$ & 12 & 1 & $?$ & 4 & $1+?$ \\
\hline 38 & 11310 & 1 & $\mathrm{~N}$ & $14+15$ & $5+10$ & 1 & 2 & 4 & 7 \\
\hline 39 & 11167 & 1 & 1 & 20 & $5+10$ & 3 & 1 & 4 & 8 \\
\hline 40 & 11188 & 1 & 1 & ? & $5+10$ & 3 & ? & 4 & $7+?$ \\
\hline 41 & $11184,11185,11187,11231$ & 4 & 1 & 9 & $5+10$ & 3 & ? & 4 & $7+?$ \\
\hline 42 & 11195 & 1 & 1 & $7 * *+9$ & $5+10$ & 3 & ? & 4 & $7+?$ \\
\hline 43 & 11199 & 1 & 1 & $7 * *+9$ & $5+10$ & 3 & $?$ & 4 & $7+?$ \\
\hline 44 & 11308 & 1 & 1 & $7(7 * *)+9$ & $5+10$ & 3 & ? & 4 & $7+?$ \\
\hline 45 & 11233 & 1 & $\mathrm{~N}$ & $7 *+9$ & $5+10$ & 1 & 2 & 4 & 7 \\
\hline 46 & 11232 & 1 & 1 & 7 & 10 & 3 & 1 & $?$ & $<10$ \\
\hline 47 & 11311 & 1 & 1 & $7+8 * *$ & $5+10$ & 3 & ? & 4 & $7+?$ \\
\hline 48 & 11298 & 1 & 1 & $7\left(7^{*}\right)+8$ & $5+10$ & 3 & 3 & 4 & 10 \\
\hline 49 & 13190 & 1 & 1 & $7+8$ & $5+10$ & 3 & 3 & 4 & 10 \\
\hline 50 & 11265 & 1 & $\mathrm{~N}$ & $7 * *+8$ & $5+10$ & 1 & ? & 4 & $5+?$ \\
\hline 51 & 11267,13192 & 2 & 1 & $7 * *+8$ & $5+10$ & 3 & $?$ & 4 & $7+?$ \\
\hline 52 & 11534 & 1 & $\mathrm{~N}$ & $7 * *+8 * *$ & $5+10$ & 1 & $?$ & 4 & $5+?$ \\
\hline 53 & $11194,11211,11332$ & 3 & 1 & $13+16$ & $5+10$ & 3 & 3 & 4 & 10 \\
\hline 54 & 11210 & 1 & $\mathrm{~N}$ & $8 *, 7+9$ & $5+10$ & 1 & ? & 4 & $5+?$ \\
\hline 55 & 11262 & 1 & $\mathrm{~N}$ & $7+9$ & $5+10$ & 1 & 2 & 4 & 7 \\
\hline 56 & 11313 & 1 & $\mathrm{~N}$ & $7(7 * *)+9$ & $5+10$ & 1 & $?$ & 4 & $5+?$ \\
\hline 57 & 11214 & 1 & $2 *$ & 9 & $5+10$ & 3 & $?$ & 4 & $7+?$ \\
\hline 58 & 11174,11176 & 2 & 1 & $8^{*}(7 *+9)$ & ? & 3 & ? & $?$ & $3+?$ \\
\hline 59 & 11288 & 1 & 1 & $7 *+9$ & 10 & 3 & $\dot{2}$ & $\dot{?}$ & $5+?$ \\
\hline 60 & 11198,11530 & 2 & 1 & $17+18$ & $5+10$ & 3 & 3 & 4 & 10 \\
\hline 61 & $11200,11202,11328$ & 3 & $\mathrm{~N}$ & $17+18$ & $5+10$ & 1 & 3 & 4 & 8 \\
\hline 62 & 11300 & 1 & $\mathrm{~N}$ & $8^{* *}(17+18)$ & $5+10$ & 1 & ? & 4 & $<10$ \\
\hline 63 & 11307 & 1 & $2 *$ & $17+18$ & $5+10$ & 3 & $\dot{3}$ & 4 & 10 \\
\hline 64 & 11527 & 1 & 1 & $6,17+18$ & $5+10$ & 3 & ? & 4 & $7+?$ \\
\hline 65 & 11303 & 1 & $\mathrm{~N}$ & $6,17+18$ & $5+12 *$ & 1 & $?$ & ? & $1+?$ \\
\hline 66 & 11538 & 1 & 1 & 17 & $5+12$ & 3 & $?$ & $?$ & $3+?$ \\
\hline
\end{tabular}

C. Commercial cultivars of bread wheat

\begin{tabular}{|c|c|c|c|c|c|c|c|c|c|}
\hline & & \multirow[t]{2}{*}{$f$} & \multicolumn{3}{|c|}{ Allelic frequency } & \multicolumn{3}{|c|}{ Quality score } & \multirow[t]{2}{*}{ Total } \\
\hline & & & Glu-Al & Glu-B1 & Glu-D1 & Glu-Al & Glu-B1 & Glu-D1 & \\
\hline 1 & $\begin{array}{l}\text { Bakhtawar 92, Zarghoon, Nowshera 96, } \\
\text { Kohsar 95, Fakhr-e-sarhad, Mehran } 89\end{array}$ & 6 & 1 & $7+9$ & $5+10$ & 3 & 2 & 4 & 9 \\
\hline 2 & Chakwal 50, Lasani 08, Fareed 06 & 3 & $\mathrm{~N}$ & $7+9$ & $5+10$ & 1 & 2 & 4 & 7 \\
\hline 3 & Watan 94 & 1 & $2 *$ & $7+9$ & $5+10$ & 3 & 2 & 4 & 9 \\
\hline 4 & Kohinoor 83 , Pak & 2 & 1 & $7 *+9$ & $5+10$ & 3 & 2 & 4 & 9 \\
\hline 5 & $\begin{array}{l}\text { Lu 26, Rohtas 90, Zardana, Moomal 2002, } \\
\text { Zariashata, GA 2002, Wafaq 01, Margalla 99, } \\
\text { Saleem 2000, Khyber 87, Pirsabak } 2004\end{array}$ & 11 & $2^{*}$ & $7 *+9$ & $5+10$ & 3 & 2 & 4 & 9 \\
\hline 6 & Darawar 97 & 1 & $2 *$ & $7 * *+9$ & $5+10$ & 3 & $?$ & 4 & $7+?$ \\
\hline 7 & Faisalabad 85 & 1 & $\mathrm{~N}$ & $17+18$ & $5+10$ & 1 & 3 & 4 & 8 \\
\hline 8 & $\begin{array}{l}\text { Parwaz 94, Seriab 92, Sarsabz, Soughat 90, } \\
\text { Tandojam 83, SH 2002, Abadgar 93, Shafaq } 2006\end{array}$ & 8 & 1 & $17+18$ & $5+10$ & 3 & 3 & 4 & 10 \\
\hline 9 & Pasban 90 & 1 & 1 & $13+16$ & $5+10$ & 3 & 3 & 4 & 10 \\
\hline 10 & Blue silver, Faisalabad 83, Bakhhar 2002, & 3 & $\mathrm{~N}$ & $7+9$ & $2+12$ & 1 & 2 & 2 & 5 \\
\hline 11 & $\begin{array}{l}\text { Kaghan 93, Shaheen 94, Shahkar 95, } \\
\text { Bahawalpur 97, Punjnad 1, V87094, Raskooh }\end{array}$ & 7 & $2 *$ & $7+9$ & $2+12$ & 3 & 2 & 2 & 7 \\
\hline 12 & MH 97 & 1 & $2^{*}$ & 7 & $2+12$ & 3 & 1 & 2 & 6 \\
\hline 13 & Kohistan 97 & 1 & $2^{*}$ & $7+9$ & $2+12$ & 3 & 2 & 2 & 7 \\
\hline 14 & Punjab 97 & 1 & $\mathrm{~N}$ & $7+8$ & $2+12$ & 1 & 3 & 2 & 6 \\
\hline
\end{tabular}




\section{Table 2. Continued.}

C. Commercial cultivars of bread wheat

\begin{tabular}{|c|c|c|c|c|c|c|c|c|c|}
\hline & & f & & llelic frequen & & & uality sco & & Total \\
\hline & & & Glu-Al & Glu-BI & $G l u-D 1$ & Glu-Al & Glu-Bl & Glu-Dl & \\
\hline 15 & Chakwal 86 & 1 & $\mathrm{~N}$ & $7^{*+8}$ & $2+12$ & 1 & 3 & 2 & 6 \\
\hline 16 & Sind 81 & 1 & $\mathrm{~N}$ & $7+8\left(8^{*}\right)$ & $2+12$ & 1 & ? & 2 & $<10$ \\
\hline 17 & Bahawalpur 2000 & 1 & $\mathrm{~N}$ & $7+8$ & $2+12$ & 1 & 3 & 2 & 6 \\
\hline 18 & Morocco & 1 & $2^{*}$ & $7+8,14$ & $2+12$ & 3 & ? & 2 & $<10$ \\
\hline 19 & Sehar 2006 & 1 & $2^{*}$ & $7+8$ & $2+12$ & 3 & 3 & 2 & 8 \\
\hline 20 & $\begin{array}{l}\text { Inqilab 91, Kirin 95, Mexipak } 65 \text {, Suleman 96, } \\
\text { WL 711, Tatara, As 2002, Iqbal 2000, Auqab 2000, } \\
\text { Pirsabak 2005, Saussi, Meraj 08, Faisalabad 08, Bathoor }\end{array}$ & 14 & $2^{*}$ & $17+18$ & $2+12$ & 3 & 3 & 2 & 8 \\
\hline 21 & Takbeer, Manthar 3 & 2 & $\mathrm{~N}$ & $17+18$ & $2+12$ & 1 & 3 & 2 & 6 \\
\hline 22 & Chakwal 97 & 1 & $\mathrm{~N}$ & $7 *(13+16)$ & 12 & 1 & ? & ? & $<10$ \\
\hline
\end{tabular}

To clarify the genetic diversity of $H M W-G S$ among wheat germplasms evaluated in the present study, separate cluster analysis of landraces from Punjab, Baluchistan, and commercial varieties was performed. Germplasms were classified into various groups and subgroups comprising single genotypes or biotypes based on allelic similarity. Two landraces from Punjab (18701 and 11361) exhibited high quality values for viscoelasticity and bread making quality. Similarly, the high quality $5+10$ subunit along with other good quality subunits were observed in 5 landraces $(11298,13190,11198,11530$, and 11307) collected from Baluchistan, which conferred 10 quality scores to these accessions. Among the commercial cultivars, the high quality allelic combination $(17+18$ and $5+10)$ was reported in 8 cultivars, including Parwaz 94, Seriab 92, Sarsabz, Soughat 90, Tandojam 83, SH-2002, Abadgar 93, and Shafaq 2006. The cultivar "Pasban 90" showed a high quality score, but the allelic combination was $13+16$ and 5+10. Thirty-four commercial cultivars of 69 contained the $5+10$ allele at the Glu-D1 locus; therefore, these have high scores regarding bread making quality. Unlike landraces, low-scoring subunits $(7,6+8,3+12$, and $2+12)$ were not found frequently in commercial cultivars. However, several good quality alleles, including $7+8,7+9$, and $5+10$, were observed in landraces of both Punjab and Baluchistan origin.

\section{DISCUSSION}

Indigenous wheat landraces and commercial cultivars possessed allelic variation/ combinations for $H M W-G S$ that indicated the uniqueness of wheat germplasms originating from Pakistan (Khalid et al., 2013). Previously, wheat landraces collected from the province of Baluchistan were reported to have unique protein subunits (Tahiru et al., 1995); thus, we evaluated the high number of landraces collected from this province to obtain a clear understanding of $H M W-G S$ diversity for documentation and for future utilization (Sajjad et al., 2012). The comparison of diversity for $H M W-G S$ among landraces collected from Punjab and Baluchistan revealed that some unique novel bands were observed in landraces originating from Baluchistan. This province has a unique agro-geographic position and is one of the centers of diversity for cereals. The abundance of the Null allele at GluAl in landraces was in stark contrast with the findings of Tabasum et al. (2011); however, these authors examined a smaller number of landraces, and genetic diversity may also be responsible for the deviation observed. Nevertheless, Lagudah et al. (1987) reported the Null allele as predominant over 1 and 2* in landraces. Elfatih et al. (2013) reported 23 new alleles at the Glu-A1 and the Glu-B1 
among 62 durum wheat accessions. Among the 242 accessions, the Glu-B1 locus displayed higher variation than did Glu-A1.

It has been reported that landraces had a larger number of polymorphisms in $H M W-G S$ than in commercial cultivars (Wang et al., 2013), which is related to the unexplored genetic potential for the wheat genome. Allelic variant $2^{*}$ was observed in a high proportion among indigenous wheat landraces, which was associated with better wheat flour quality (Laidò et al., 2013). More than half of the commercial cultivars carried this allele, indicating that breeders rigorously selected wheat cultivars with better quality that were indirectly coupled to the $2^{*}$ allele (He et al., 2013). Ribeiro et al. (2013) analyzed the Portuguese wheat landrace 'Barbela' and reported a new $x$-type $H M W$-GS encoded at the Glu-A1 locus that was named 1Ax1.1. Lan et al. (2013) reported low-molecular weight glutenin subunits as the major components of glutenins, which were considered to be important for the end-use quality of wheat; however, Kaur et al. (2013) reported the physico-chemical and rheological properties of Indian wheat cultivars varying in $H M W-G S$. Pompa et al. (2013) reported that the gluten composition and expression influenced dough properties and were cultivar- and environment-dependent. Additionally, the cultivar Simeto was the most stable across environments.

The maximum variation in the number of both alleles and polymorphisms was observed at the $G l u-B 1$ locus, particularly among landraces collected from Baluchistan. This has also been reported previously by McIntosh et al. (2003), who identified 56 alleles in Glu-B1, whereas Sultana et al. (2007), who examined the genetic variability of Pakistani wheat based on polymorphisms in $H M W-G S$, reported 3 allelic variants (Null, 1 and 2*) at the Glu-A1 locus, $9\left[7+8,7^{*}+8,7\left(7^{*}\right)+8,7+9,7^{*}+9,14,13+16,17+18\right.$, and 20$]$ at the Glu-B1 locus, and 3 allelic variants $\left(5+10,2+12\right.$, and $2^{\left.* *+12^{\prime}\right]}$ at the Glu-D1 locus in accordance with the descriptions of Payne and Lawrence (1983) and Marchylo et al. (1992). These results were in agreement with our studies; however, allele $2^{* *+12^{\prime}}$ was not present in our germplasm. A large number of rare alleles was reported, which were confirmed by the findings of Branlard (2003), Tabasum (2011), and Dessalegn (2011). Several novel HMW-GS alleles, such as 7*, $7^{* *}, 8^{*}$, and $8^{* *}$ (Liu et al., 2007), were detected in the present study and have also been detected in Chinese wheat landraces.

The novel alleles observed in the landraces, particularly those collected from Baluchistan, may have formed following the introduction of rare mutations (Costa et al., 2013). As this material was not previously evaluated for $H M W-G S$, determining the quality score of these subunits is important (Goutam et al., 2013). The low quality scores of the Pakistani commercial wheat varieties are in accordance with the expectations, as most breeding programs involving wheat in the country have focused on yield improvement (Zhong-hu et al. 1992). The present results indicate that the development of commercial varieties has reduced the genetic diversity of wheat in terms of $H M W-G S$, as lower genetic diversity in commercial varieties was observed. However, this is mainly because of the selection pressure for economic traits, including yield and quality. This result is consistent with those of previous studies by Atanasova et al. (2009) and Morgunov et al. (1993). These findings are in contrast to those of Hirano et al. (2008), who showed that the introduction of high-yielding varieties did not reduce the genetic variability of landraces of wheat in Pakistan. However, Hirano et al. (2008) studied a small population of landraces, which was not sufficient to draw a valid conclusion. In addition to the presence of high quality allele $5+10$ in local landraces, other favorable alleles were identified in the Baluchistan and Punjab accessions. Because most wheat breeding programs used $5+10$, subunits $17+18,13+16$, and $14+15$ should also be used, as they have a 
positive influence on the grain quality indices of wheat. As a result, genetic diversity as well as end-use quality would improve (Liu et al., 2007). The exploration of diversity in available genetic stocks and quality associated with favorable alleles is important, and can be effectively utilized to develop new cultivars and improve crops.

\section{REFERENCES}

Ahmad M, Shahzad A, Iqbal M, Asif M, et al. (2013). Morphological and molecular genetic variation in wheat for salinity tolerance at germination and early seedling stage. Aust. J. Crop Sci. 7: 66-74.

Atanasova D, Tsenov N, Todorov I and Ivanova I (2009). Glutenin composition of winter wheat varieties bred in Dobrudzha Agricultural Institute. Bulgarian J. Agric. Sci. 15: 9-19.

Branlard G, Dardevet M, Amiour N and Igrejas G (2003). Allelic diversity of HMW and LMW glutenin subunits and omegagliadins in French bread wheat (Triticum aestivum L.). Genet. Resour. Crop Evol. 50: 669-679.

Costa MS, dos Santos-Scholz MB and Franco CML (2013). Effect of high and low molecular weight glutenin subunits, and subunits of gliadin on physicochemical parameters of different wheat genotypes. Cienc. Technol. Aliment Campinas 33: 163-170.

Degaonkar AM, Tamhankar SA and Rao VS (2005). An assessment of cultivated emmer germplasm for gluten proteins. Euphytica 145: 49-55.

Dessalegn T, Van deventer CS, Labuschagne MT and Martens H (2011). Allelic variation of HMW glutenin subunits of Ethiopian bread wheat cultivars and their quality. Afr. Crop Sci. J. 19: 55-63.

Elfatih SE, Peng Y, Ma J, Peng J, et al. (2013). High frequency of unusual high molecular weight glutenin alleles in 232 tetraploid durum wheat accessions (Triticum turgidum L. ssp. durum Desf). Cereal Res. Comm. 41: 583-592.

Goutam U, Kukreja S, Tiwari R, Chaudhury A, et al. (2013). Biotechnological approaches for grain quality improvement in wheat: present status and future possibilities. Aust. J. Crop Sci. 7:469-483.

He J, Penson S, Powers SJ, Hawes C, et al. (2013). Spatial patterns of gluten protein and polymer distribution in wheat grain. J. Agric. Food Chem. 61: 6207-6215.

Hirano R, Kikuchi A, Kawase M and Watanabe KN (2008). Evaluation of genetic diversity of bread wheat landraces from Pakistan by AFLP and implications for a future collection strategy. Genet. Resour. Crop Evol. 5: 1007-1015.

Kaur A, Singh N, Ahlawat AK, Kaur S, et al. (2013). Diversity in grain, flour, dough and gluten properties amongst Indian wheat cultivars varying in high molecular weight subunits (HMW-GS). Food Res. Int. 53: 63-72.

Khalid M, Mahmood T, Rasheed A, Kazi AG, et al. (2013). GLU-D1 allelic variation in synthetic hexaploid wheat derived from durum cultivar 'Decoy x Aegilops tauschii accessional crosses. Pak. J. Bot. 45: 409-414.

Khan J, Khan S, Khetran MA, Amanullah, et al. (2013). Tijaban-10 a drought tolerant and high yielding wheat variety of rainfed/sailaba areas of Baluchistan. Pak. J. Bot. 45: 1357-1362.

Lagudah ES, Flood RG and Halloran GM (1987). Variation in high molecular weight glutenin sub-units in land races of hexaploid wheat from Afghanistan. Euphytica 36: 3-9.

Laidò G, Mangini G, Taranto F, Gadaleta A, et al. (2013). Genetic diversity and population structure of tetraploid wheats (Triticum turgidum L.) estimated by SSR, DArT and pedigree data. PLoS One 8: e67280.

Lan Q, Feng B, Xu Z, Zhao G, et al. (2013). Molecular cloning and characterization of five novel low molecular weight glutenin subunit genes from Tibetan wheat landraces (Triticum aestivum L.). Genet. Resour. Crop Evol. 60: 799-806.

Li Vigni M, Baschieri C, Marchetti A and Cocchi M (2013). RP-HPLC and chemometrics for wheat flour protein characterisation in an industrial bread-making process monitoring context. Food Chem. 139: 553-562.

Li Y, Ye W, Wang M and Yan X (2009). Climate change and drought: a risk assessment of crop yield impacts. Clim. Res. 39: 31-46.

Liu L, He ZH, Yan J, Zhang Y, et al. (2005). Allelic variation at the Glu-1 and Glu-3 loci, presence of 1B/1R translocation, and their effect on mixgraphic properties in Chinese bread wheats. Euphytica 142: 197-204.

Liu Y, Xiong ZY, He YG, Shewry PR, et al. (2007). Genetic diversity of HMW-GS in Chinese common wheat (Triticum aestivum L.) landraces from Hubei province. Genet. Resour. Crop. Evol. 54: 865-874.

Marchylo BA, Lukow OM and Kruger JE (1992). Quantitative variation in high molecular weight glutenin subunit 7 in some Canadian wheats. J. Cereal Sci. 15: 29-37.

McIntosh RA, Yamazaki Y, Devos KM, Dubcovsky J, et al. (2003). Catalogue of gene symbols for wheat. Available at [http://wheat.pw.usda.gov/ggpages/wgc]. Accessed March 3, 2011.

Morgunov AI, Pena RJ, Crossa J and Rajaram S (1993). Worldwide distribution of Glu-1 alleles in bread wheat. J. Genet. Breed. 47: 53-60. 
Payne PI (1987). Genetics of wheat storage proteins and the effect of allelic variation on bread-making quality. Annu. Rev. Plant Physiol. 38: 141-153.

Payne PI and Lawrence GJ (1983). Catalogue of alleles for the complex loci, Glu-A1, Glu- B1 and Glu-D1, which code for high molecular-weight subunits of glutenin in hexaploid wheat. Cereal Res .Commun. 11: 29-35.

Peter R, Shewry N and Halford G (2002). Cereal seed storage proteins: structures, properties and role in grain utilization. IACR-Long Ashton Research Station, Department of Agricultural Sciences, University of Bristol, Long Ashton, Bristol.

Pompa M, Giuliani MM, Palermo C, Agriesti F, et al. (2013). Comparative analysis of gluten proteins in three durum wheat cultivars by a proteomic approach. J. Agric. Food Chem. 61: 2606-2617.

Redaelli R, Pogna NE and Ng PKW (1997). Effects of prolamins encoded by chromosomes 1B and 1D on the rheological properties of dough in near-isogenic lines of bread wheat. Cereal Chem. 74: 102-107.

Ribeiro M, Bancel E, Faye A, Dardevet M, et al. (2013). Proteogenomic characterization of novel x-type high molecular weight glutenin subunit 1Ax1.1. Int. J. Mol. Sci. 14: 5650-5667.

Sajjad M, Khan SH, Maqbool R, Ather A, et al. (2012). Selection of Pakistani and CIMMYT wheat lines for better grain yield and quality. Int. J. Agric. Biol. 14: 645-649

Sultana T, Ghafoor A and Ashraf M (2007). Genetic variability in bread wheat (Triticum aestivum L.) of Pakistan based on polymorphism for high molecular weight glutenin subunits. Genet. Resour. Crop Evol. 54: 1159-1165.

Tabasum A, Iqbal N, Hameed A and Arshad R (2011). Evaluation of Pakistani wheat germplasm for bread quality based on allelic variation in $H M W$ glutenin subunits. Pak. J. Bot. 43: 1735-1740.

Tahiru M, Hussain SA, Turchetta T and Lafiandram D (1995). The HMW glutenin subunit composition of bread wheat varieties of Pakistan. Plant Breed. 114: 442-444.

Wang S, Yu Z, Cao M, Shen X, et al. (2013). Molecular mechanisms of HMW glutenin subunits from 1S(I) genome of Aegilops longissima positively affecting wheat bread making quality. PloS One 8: e58947.

Yan YM, Jiang Y, An XL, Pei YH, et al. (2009). Cloning, expression and functional analysis of HMW glutenin subunit 1 By8 gene from Italy pasta wheat (Triticum turgidum L. ssp. Durum). J. Cereal Sci. 50: 398-406.

Yu Z, Han C, Yan X, Li X, et al. (2013). Rapid characterization of wheat low molecular weight glutenin subunits by ultraperformance liquid chromatography (UPLC). J. Agric. Food Chem. 61: 4026-4034.

Žilić S, Barać M, Pešić M, Dodig D, et al. (2011). Characterization of proteins from grain of different bread and durum wheat genotypes. Int. J. Mol. Sci. 12: 5878-5894.

Zhong-hu H, Peña RJ and Rajaram S (1992). High molecular weight glutenin subunit composition of Chinese bread wheats. Euphytica 64: 11-20. 